\title{
Multipliers on vector valued Bergman spaces
}

\author{
Oscar Blasco, José Luis Arregui*
}

\begin{abstract}
Let $X$ be a complex Banach space and let $B_{p}(X)$ denote the vector-valued Bergman space on the unit disc for $1 \leq p<\infty$. A sequence $\left(T_{n}\right)_{n}$ of bounded operators between two Banach spaces $X$ and $Y$ defines a multiplier between $B_{p}(X)$ and $B_{q}(Y)$ (respect. $B_{p}(X)$ and $\ell_{q}(Y)$ ) if for any function $f(z)=\sum_{n=0}^{\infty} x_{n} z^{n}$ in $B_{p}(X)$ we have that $g(z)=\sum_{n=0}^{\infty} T_{n}\left(x_{n}\right) z^{n}$ belongs to $B_{q}(Y)$ (respect. $\left(T_{n}\left(x_{n}\right)\right)_{n} \in$ $\left.\ell_{q}(Y)\right)$. Several results on these multipliers are obtained, some of them depending upon the Fourier or Rademacher type of the spaces $X$ and $Y$. New properties defined by the vector-valued version of certain inequalities for Taylor coefficients of functions in $B_{p}(X)$ are introduced.
\end{abstract}

\section{Introduction.}

Operator valued multipliers between spaces of vector-valued functions have been recently considered by different authors and for different reasons (see [32], [22], [1] and [10]). In particular, a big effort has been done in the case of spaces of vector-valued analytic functions such as BMOA, Bloch and Hardy spaces (see [9], [10] and [13]). In this paper we shall study multipliers on Bergman spaces in the vector-valued situation.

Given $1 \leq p<\infty$ any complex Banach space $X$ we shall use the notation $B_{p}(X)$ for the space of $X$-valued analytic functions $f: \mathbb{D} \rightarrow X$ defined on the unit disc $\mathbb{D}$ which are $p$-integrable against the Lebesgue measure in the disc $\mathbb{D}$ denoted by $d A(z)$. We shall use the norm

$$
\|f\|_{B_{p}(X)}=\left(\int_{0}^{1} M_{p}^{p}(f, r) d r\right)^{1 / p}
$$

\footnotetext{
*Both authors have been partially supported by Proyecto PB98-0146
} 
where $M_{p}(f, r)=\left(\int_{0}^{2 \pi}\left\|f\left(r e^{i t}\right)\right\|^{p} \frac{d t}{2 \pi}\right)^{1 / p}$.

Let $1 \leq p, q<\infty$ and let $X$ and $Y$ be complex Banach spaces, a sequence of bounded operators $\left(T_{n}\right)_{n}$ in $\mathcal{L}(X, Y)$ is said to be a multiplier between $B_{p}(X)$ and $B_{q}(Y)$, to be denoted $\left(T_{n}\right) \in\left(B_{p}(X), B_{q}(Y)\right)$, if for any function $f(z)=\sum_{n=0}^{\infty} x_{n} z^{n}$ in $B_{p}(X)$ we have that $g(z)=\sum_{n=0}^{\infty} T_{n}\left(x_{n}\right) z^{n}$ belongs to $B_{q}(Y)$.

A closed graph argument shows this to be equivalent to the existence of a constant $C>0$ such that

$$
\left\|\sum_{n=0}^{N} T_{n}\left(x_{n}\right) z^{n}\right\|_{B_{q}(Y)} \leq C\left\|\sum_{n=0}^{N} x_{n} z^{n}\right\|_{B_{q}(X)}
$$

for any $N \in \mathbb{N}$ and $x_{1}, x_{2}, \ldots, x_{N}$ elements in $X$.

We shall be denoting by $\ell_{p}(X)$ the space of sequences $\left(x_{n}\right)_{n}$ in $X$ such that $\left\|\left(x_{n}\right)\right\|_{p}=\left(\sum_{n=0}^{\infty}\left\|x_{n}\right\|^{p}\right)^{1 / p}<\infty$. As above, a sequence of bounded operators $\left(T_{n}\right)_{n}$ in $\mathcal{L}(X, Y)$ is said to be a multiplier between $B_{p}(X)$ and $\ell_{q}(Y)$, to be denoted $\left(T_{n}\right) \in\left(B_{p}(X), \ell_{q}(Y)\right)$, if for any function $f(z)=\sum_{n=0}^{\infty} x_{n} z^{n}$ in $B_{p}(X)$ we have that the sequence $\left(T_{n}\left(x_{n}\right)\right)$ belongs to $\ell_{q}(Y)$.

Again this is equivalent to the existence of a constant $C>0$ such that

$$
\left(\sum_{n=0}^{N}\left\|T_{n}\left(x_{n}\right)\right\|^{q}\right)^{1 / q} \leq C\left\|\sum_{n=0}^{N} x_{n} z^{n}\right\|_{B_{q}(X)}
$$

for any $N \in \mathbb{N}$ and $x_{0}, x_{1}, \ldots, x_{N}$ elements in $X$.

The infimum of the constants $C$ verifying (1) or (2) is the multiplier norm, which coincides with the operator norm between $B_{p}(X)$ and $B_{q}(Y)$ or $\ell_{q}(Y)$ respectively.

We shall try to give necessary and sufficient conditions for a sequence $\left(T_{n}\right)$ to be multiplier in $\left(B_{p}(X), B_{q}(Y)\right.$ or $\left(B_{p}(X), \ell_{q}(Y)\right)$.

In the scalar-valued case the complete characterization in known in many cases. The reader is referred to [11] and [25] for a recollection of the old and new results about multipliers for spaces of analytic functions, which, in particular, apply to Bergman spaces. We list here those which are completely described and which will be studied later on.

If $1 \leq q \leq \infty$ then (see [18])

$$
\left(B_{1}, \ell_{q}\right)=\left\{\left(\lambda_{n}\right)_{n}: \sup _{k \geq 0} 2^{k}\left(\sum_{n=2^{k}+1}^{2^{k+1}}\left|\lambda_{n}\right|^{q}\right)^{1 / q}<\infty\right\}
$$


The reader is also referred to [7] or [11] for a proof obtained from a very general principle of operators acting on $B_{1}$.

If $2 \leq p<\infty, 1 \leq q \leq \infty, 1 / s=1 / \min (q, 2)-1 / 2$ and $1 / r=$ $1 / \min (p, q)-1 / p$ then (see [28], [11] or [25])

$$
\left(B_{p}, \ell_{q}\right)=\left\{\lambda_{n}:\left(\sum_{k \geq 0} 2^{k r / p}\left(\sum_{n=2^{k}+1}^{2^{k+1}}\left|\lambda_{n}\right|^{s}\right)^{r / s}\right)^{1 / r}<\infty\right\}
$$

If $1 \leq q \leq \infty$ then (see [25], [7] or [11])

$$
\left(B_{1}, B_{q}\right)=\left\{\lambda(z)=\sum_{n=0}^{\infty} \lambda_{n} z^{n}: \sup _{0<r<1}(1-r)^{1 / q} M_{q}\left(\lambda^{\prime}, r\right)<\infty\right\}
$$

If $1 \leq q \leq 2 \leq p<\infty$ and $1 / r=1 / q-1 / p$ then (see [33])

$$
\left(B_{p}, B_{q}\right)=\left\{\lambda_{n}:\left(\sum_{k \geq 0} 2^{-k} \sup _{2^{k}+1 \leq n<2^{k+1}}\left|\lambda_{n}\right|^{r}\right)^{1 / r}<\infty\right\}
$$

Some alternative proofs of (6) were given independently in [2], [8] and [24].

The conditions appearing in (3), (4) and (6) can be formulated in terms of certain mixed norm sequence spaces, denoted by $\ell(p, q)$, consisting of sequences $\left(\lambda_{n}\right)$ such that $\|\left.\left(\lambda_{n}\right)\right|_{p, q}=\left(\sum_{k \geq 0}\left(\sum_{n=2^{k}+1}^{2^{k+1}}\left|\lambda_{n}\right|^{p}\right)^{q / p}\right)^{1 / q}<\infty$. Also it is known that the condition apperaring in (5) is equivalent to the Lipschizt condition $\left\|\Delta_{t} \lambda\right\|_{q}=O\left(|t|^{1 / q^{\prime}}\right)$ (see Section 5).

The paper is divided into four sections. The first one is devoted to introduce and prove some results on the mixed norm spaces $\ell(p, q, X)$. It is shown that $\left(\ell\left(p_{1}, q_{1}, X\right), \ell\left(p_{2}, q_{2}, Y\right)\right)=\ell(p, q, \mathcal{L}(X, Y))$ where $1 / p=\left(\left(1 / p_{2}\right)-\right.$ $\left.\left(1 / p_{1}\right)\right)^{+}$and $1 / q=\left(\left(1 / q_{2}\right)-\left(1 / q_{1}\right)\right)^{+}$and some reformulations for the norms in $\ell(p, q)$ are given.

In Section 3 we prove some results on Taylor coefficients of vector valued functions in $B_{p}(X)$. We introduce the properties of Bergman type and cotype. Given $1 \leq p \leq 2 \leq q \leq \infty$, a Banach space $X$ is said to have Bergman type $p$ or Bergman cotype $q$ if there exists a $C>0$ such that

$$
\left\|\left(\frac{x_{n}}{n^{1 / p}}\right)_{1 \leq n \leq N}\right\|_{p^{\prime}, p} \leq C\|f\|_{B_{p}(X)}
$$


or

$$
\|f\|_{B_{q}(X)} \leq C\left\|\left(\frac{x_{n}}{n^{1 / q}}\right)_{1 \leq n \leq N}\right\|_{q^{\prime}, q}
$$

for all $N \in \mathbb{N}, x_{1}, \ldots, x_{N}$ in $X$ and $f(z)=\sum_{n=0}^{N} x_{n} z^{n}$ respectively.

It is shown that, for $1 \leq p \leq 2$, spaces of Fourier type $p$ must also have Bergman type $p$ and Bergman cotype $p^{\prime}$ where $1 / p+1 / p^{\prime}=1$.

Section 4 is devoted to analyze the extension of (3) and (4) to the vector valued setting, showing that the result for $B_{1}(X)$ has a natural extension with no condition on the Banach spaces, while each of the embeddings in (4) relies upon certain geometric properties on the spaces such as the Bergman, Rademacher or Fourier type.

Finally in Section 5 we deal with the vector-valued interpretation of (5) and (6). Again a version of (5), and some equivalent description in terms of Lipchizt classes, is obtained without conditions on $X$ and $Y$. Also the embeddings of the corresponding result for (6) can only be proved under some assumptions on $X$ and $Y$. In particular (6) holds for operators $\left(T_{n}\right) \subset$ $\mathcal{L}(X, Y)$ between Hilbert spaces $X$ and $Y$.

Throughout the paper $C$ denotes a constant that may vary from line to line, $\alpha^{+}=\max \{\alpha, 0\}$ for $\alpha \in \mathbb{R}$ and $p^{\prime}$ stands for the conjugate exponent of $p$, i.e. $1 / p+1 / p^{\prime}=1$.

\section{Mixed norm sequences spaces.}

Let us now introduce a family of sequence vector spaces with mixed norms, in terms of which many on the results on multipliers in the following sections will be obtained.

De fition 2.1 Let $1 \leq p, q \leq \infty$, and let Banach $X$ be a Banach space. $\ell(p, q, X)$ denotes the space of sequences $\left(x_{n}\right)_{n} \subset X$ such that $\left(\left\|\left(x_{n}\right)_{n \in I_{k}}\right\|_{\ell_{p}}\right)_{k} \in$ $\ell_{q}$, where $I_{k}=\left\{n \in \mathbb{N} ; 2^{k-1} \leq n<2^{k}\right\}$ for $k \in \mathbb{N}$ and $I_{0}=\{0\}$.

For $1 \leq p, q<\leq \infty$, the spaces $\ell(p, q, X)$ become a Banach space under the norm $\|\cdot\|_{p, q}$ given by

$$
\left\|\left(x_{n}\right)\right\|_{p, q}=\left(\sum_{k=0}^{\infty}\left(\sum_{n \in I_{k}}\left\|x_{n}\right\|^{p}\right)^{q / p}\right)^{1 / q}
$$

with the obvious modifications for $q=\infty$ or $p=\infty$. 
As usual, when $X=\mathbb{C}$ we simply write $\ell(p, q)$.

Let us prove the vector-valued extension of the characterization of multipliers between $\ell(p, q)$ spaces proved in [26]. First we show the following extension of Hölder's inequality.

Proposition 2.2 Let $X$ and $Y$ be Banach spaces, $1 \leq p_{1}, p_{2} \leq \infty$ and $p$ such that $1 / p=\left(\left(1 / p_{2}\right)-\left(1 / p_{1}\right)\right)^{+}$. Then

$$
\left(\ell_{p_{1}}(X), \ell_{p_{2}}(Y)\right)=\ell_{p}(\mathcal{L}(X, Y))
$$

with equality of norms.

PROOF. Using $\left\|T_{n}\left(x_{n}\right)\right\| \leq\left\|T_{n}\right\| .\left\|x_{n}\right\|$ and Hölder's inequality one obtains the embedding $\ell_{p}(\mathcal{L}(X, Y)) \subset\left(\ell_{p_{1}}(X), \ell_{p_{2}}(Y)\right)$.

For the other embedding, let us take $\left(T_{n}\right) \in\left(\ell_{p_{1}}(X), \ell_{p_{2}}(Y)\right)$ with norm 1. Hence for any $\left(\alpha_{n}\right) \in \ell_{p_{1}}$ and any $\left(x_{n}\right)$ in the unit ball of $X$ one has that $T_{n}\left(\alpha_{n} x_{n}\right) \in \ell_{p_{2}}(Y)$ and

$$
\left\|\left(T_{n}\left(\alpha_{n} x_{n}\right)\right)\right\|_{\ell_{p_{2}}(Y)} \leq\left\|\left(\alpha_{n}\right)\right\|_{\ell_{p_{1}}} .
$$

Now, given $\varepsilon>0$, choosing $\left(x_{n}\right)$ in the unit ball of $X$ such that $\left\|T_{n}\right\|<$ $\left\|T_{n}\left(x_{n}\right)\right\|+\varepsilon / 2^{n}$ one gets that

$$
\left\|\left(\| T_{n}|| \cdot\left|\alpha_{n}\right|\right)\right\|_{\ell_{p_{2}}} \leq\left\|\left(\alpha_{n}\right)\right\|_{\ell_{p_{1}}}+C \varepsilon .
$$

This gives that $\left(T_{n}\right) \in \ell_{p}(\mathcal{L}(X, Y))$ and $\sum_{n=0}^{\infty}\left\|T_{n}\right\|^{p} \leq 1$.

Theorem 2.3 Let $X$ and $Y$ be Banach spaces and $1 \leq p_{1}, p_{2}, q_{1}, q_{2} \leq \infty$. Then

$$
\left(\ell\left(p_{1}, q_{1}, X\right), \ell\left(p_{2}, q_{2}, Y\right)\right)=\ell(p, q, \mathcal{L}(X, Y))
$$

with equality of norms, where $1 / p=\left(\left(1 / p_{2}\right)-\left(1 / p_{1}\right)\right)^{+}$and $1 / q=\left(\left(1 / q_{2}\right)-\right.$ $\left.\left(1 / q_{1}\right)\right)^{+}$.

PROOF. Let $\left(T_{n}\right)_{n}$ and $\left(x_{n}\right)_{n}$ be sequences in $\mathcal{L}(X, Y)$ and $\ell\left(p_{1}, q_{1}, X\right)$. Applying Hölder's inequality twice, one has for any $k \in \mathbb{N}$

$$
\left\|\left(T_{n} x_{n}\right)_{n \in I_{k}}\right\|_{p_{2}} \leq\left\|\left(T_{n}\right)_{n \in I_{k}}\right\|_{p}\left\|\left(x_{n}\right)_{n \in I_{k}}\right\|_{p_{1}}
$$


and that

$$
\left\|\left(T_{n} x_{n}\right)\right\|_{p_{2}, q_{2}} \leq\left\|\left(T_{n}\right)\right\|_{p, q}\left\|\left(x_{n}\right)\right\|_{p_{1}, q_{1}} .
$$

Hence $\ell(p, q, \mathcal{L}(X, Y))$ isometrically embedds into $\left(\ell\left(p_{1}, q_{1}, X\right), \ell\left(p_{2}, q_{2}, Y\right)\right)$.

Conversely, let $\left(T_{n}\right)$ be a multiplier in $\left(\ell\left(p_{1}, q_{1}, X\right), \ell\left(p_{2}, q_{2}, Y\right)\right)$ and let us write $\beta_{k}=\left\|\left(T_{n}\right)_{n \in I_{k}}\right\|_{p}$.

Given $N \in \mathbb{N}$ we may choose a family of nonnegative numbers $\left(\alpha_{k}\right)_{1 \leq k \leq N}$ with norm in $\ell_{q_{1}}$ equal to 1 and such that $\left\|\left(\beta_{k}\right)_{1 \leq k \leq N}\right\|_{q}=\left\|\left(\alpha_{k} \beta_{k}\right)_{1 \leq k \leq N}\right\|_{q_{2}}$.

Now, for each $k \leq N$ we take a family of vectors $\left(x_{n}\right)_{n \in I_{k}} \subset X$ with norm in $\ell_{p_{1}}(X)$ equalling $\alpha_{k}$ and with $\left\|\left(T_{n} x_{n}\right)_{n \in I_{k}}\right\|_{p_{2}}$ arbitrarily close to $\alpha_{k} \beta_{k}$.

The norm in $\ell_{q_{2}}$ of the sequence $\left(\left\|\left(T_{n} x_{n}\right)_{n \in I_{k}}\right\|_{p_{2}}\right)_{1 \leq k \leq N}$ is then as close as we wish to $\left\|\left(\alpha_{k} \beta_{k}\right)_{1 \leq k \leq N}\right\|_{q_{2}}=\left\|\left(\beta_{k}\right)_{1 \leq k \leq N}\right\|_{q}$, necessarily less or equal to $\left\|\left(T_{n}\right)_{1 \leq n<2^{N}}\right\|_{\left(\ell\left(p_{1}, q_{1}, X\right), \ell\left(p_{2}, q_{2}, Y\right)\right)}\left\|\left(x_{n}\right)_{1 \leq n<2^{N}}\right\|_{p_{1}, q_{1}}$.

Therefore for every $N$ it holds that

$$
\left\|\left(\beta_{k}\right)_{1 \leq k \leq N}\right\|_{q} \leq\left\|\left(T_{n}\right)_{1 \leq n<2^{N}}\right\|_{\left(\ell\left(p_{1}, q_{1}, X\right), \ell\left(p_{2}, q_{2}, Y\right)\right)}
$$

as we wanted.

Now we list some useful reformulations for the norms in the spaces $\ell(p, q)$.

Lemma 2.4 (see [16] or [7]) Let $\left(\alpha_{n}\right)$ be a sequence of nonnegative numbers and $\beta>0$. Then $\sum_{n=1}^{\infty} \alpha_{n} r^{n}=O\left(\left(\frac{1}{1-r}\right)^{\beta}\right) \quad(r \rightarrow 1)$ if and only if $\left(\frac{\alpha_{n}}{n^{\beta}}\right) \in$ $\ell(1, \infty)$.

Lemma 2.5 Let $\left(\alpha_{n}\right)$ be sequence of nonnegative numbers and $\beta>0$. Then $\sup _{n} \alpha_{n} r^{n}=O\left(\frac{1}{(1-r)^{\beta}}\right) \quad(r \rightarrow 1)$ if and only if $\left(\frac{\alpha_{n}}{n^{\beta}}\right) \in \ell_{\infty}$.

PROOF. The function $r \mapsto r^{n}(1-r)^{\beta}$ attains its maximum at $n / n+\beta$ and $(n / n+\beta)^{n}(\beta / n+\beta)^{\beta} \leq C / n^{\beta}$. Therefore

$$
(1-r)^{\beta} \sup _{n} \alpha_{n} r^{n} \leq C \sup _{n} \frac{\alpha_{n}}{n^{\beta}} .
$$

The converse follows by taking $r=1-1 / n$. 
Lemma 2.6 (see [11] or [28]) Let $\left(\alpha_{n}\right)$ be sequence of nonnegative numbers and $0<q, \beta<\infty$. Then

$$
\int_{0}^{1}(1-r)^{\beta q-1}\left(\sum_{n=1}^{\infty} \alpha_{n} r^{n}\right)^{q} d r \sim \sum_{k=1}^{\infty}\left(\sum_{n \in I_{k}} \frac{\alpha_{n}}{n^{\beta}}\right)^{q} .
$$

In particular, $\left(\frac{\alpha_{n}}{n^{1 / q}}\right) \in \ell(1, q)$ if and only if $\sum_{n=1}^{\infty} \alpha_{n} r^{n} \in L_{q}((0,1), d r)$.

\section{Taylor coefficients of functions in $B_{p}(X)$.}

If $f(z)=\sum_{n=0}^{\infty} x_{n} z^{n}$ with $x_{n} \in X$ then, for each $n$ and $r \in(0,1)$, we have that

$$
x_{n} r^{n}=\frac{1}{2 \pi} \int_{-\pi}^{\pi} f\left(r e^{i \theta}\right) e^{-i n \theta} d \theta .
$$

This implies that for any $n \in \mathbb{N}$ and $0<r<1$ we have

$$
\left\|x_{n}\right\| r^{n} \leq M_{1}(f, r)
$$

This simple observation allows us to get the following results on Taylor coefficients of functions in Bergman spaces.

Proposition 3.1 Let $1 \leq p<\infty$ and $f(z)=\sum_{n=0}^{\infty} x_{n} z^{n}$. If $f \in B_{p}(X)$ then $\left\|x_{n}\right\|=o\left(n^{1 / p}\right)$ and $\sum_{n=1}^{\infty} \frac{\left\|x_{n}\right\|^{p}}{n^{2}}<\infty$.

PROOF. Since $M_{p}(f, \cdot)$ is increasing in $(0,1)$, from $(7)$ one gets that

$$
(1-r)\left\|x_{n}\right\|^{p} r^{n p} \leq(1-r) M_{p}^{p}(f, r) \leq \int_{r}^{1} M_{p}^{p}(f, s) d s
$$

for each $r \in(0,1)$.

Hence, for any $n$, by taking $r=1-1 / n$, we see that

$$
\frac{1}{n}\left\|x_{n}\right\|^{p} \sim \frac{1}{n}\left\|x_{n}\right\|^{p}\left(1-\frac{1}{n}\right)^{n p} \leq \int_{1-1 / n}^{1} M_{p}^{p}(f, s) d s .
$$

This shows that $\frac{\left\|x_{n}\right\|}{n^{1 / p}} \rightarrow 0$. 
Now observe that the norm in $B_{p}(X)$ can be estimated from below as follows

$$
\begin{aligned}
\sum_{n=1}^{\infty} \int_{1-1 / n}^{1-1 /(n+1)} M_{p}^{p}(f, r) d r & \geq \sum_{n=1}^{\infty} \int_{1-1 / n}^{1-1 /(n+1)}\left\|x_{n}\right\|^{p} r^{n p} d r \\
& \geq C \sum_{n=1}^{\infty}\left\|x_{n}\right\|^{p} \frac{1}{n(n+1)}\left(1-\frac{1}{n}\right)^{n p} \\
& \sim \sum_{n=1}^{\infty} \frac{\left\|x_{n}\right\|^{p}}{n^{2}}
\end{aligned}
$$

Let us get an improvement of Proposition 3.1 by making use of the spaces $\ell(p, q, X)$.

Theorem 3.2 Let $1 \leq p<\infty$. There exist $C_{1}, C_{2}>0$ such that

$$
C_{1}\left\|\left(\frac{x_{n}}{n^{1 / p}}\right)\right\|_{\infty, p} \leq\|f\|_{B_{p}(X)} \leq C_{2}\left\|\left(\frac{x_{n}}{n^{1 / p}}\right)\right\|_{1, p}
$$

for any $f \in B_{p}(X)$ with Taylor coefficients $\left(x_{n}\right)$.

PROOF. Since $\|f\|_{B_{p}(X)} \leq\left(\int_{0}^{1} M_{\infty}^{p}(f, r) d r\right)^{1 / p}$ and $M_{\infty}(f, r) \leq \sum_{n=0}^{\infty}\left\|x_{n}\right\| r^{n}$ then Lemma 2.6 implies

$$
\|f\|_{B_{p}(X)} \leq\left(\int_{0}^{1}\left(\sum_{n=0}^{\infty}\left\|x_{n}\right\| r^{n}\right)^{p} d r\right)^{1 / p} \leq C\left\|\left(\frac{x_{n}}{n^{1 / p}}\right)\right\|_{1, p} .
$$

For the other inequatily, we observe that

$$
\begin{aligned}
\int_{0}^{1} M_{p}^{p}(f, r) d r & \geq \sum_{k=0}^{\infty} \int_{1-2^{-k}}^{1-2^{-(k+1)}} M_{p}^{p}(f, r) d r \\
& \geq \sum_{k=0}^{\infty} \int_{1-2^{-k}}^{1-2^{-(k+1)}}\left(\sup _{n \in I_{k}} r^{n p}\left\|x_{n}\right\|^{p}\right) d r \\
& \geq \sum_{k=0}^{\infty}\left(1-2^{-k}\right)^{p 2^{k+1}} 2^{-(k+1)} \sup _{n \in I_{k}}\left\|x_{n}\right\|^{p}
\end{aligned}
$$


Therefore

$$
\|f\|_{B_{p}(X)}^{p} \geq \sum_{k=0}^{\infty} 2^{-k} \sup _{n \in I_{k}}\left\|x_{n}\right\|^{p} \sim \sum_{k=0}^{\infty}\left(\sup _{n \in I_{k}} \frac{\left\|x_{n}\right\|^{p}}{n}\right) \sim\left\|\left(\frac{x_{n}}{n^{1 / p}}\right)\right\|_{\infty, p}^{p} .
$$

We can now get the following corollary for lacunary functions..

Corollary 3.3 (see [28] or [12]) Let $1 \leq p<\infty$ and $f(z)=\sum_{n=0}^{\infty} x_{n} z^{2^{n}}$. Then

$$
\|f\|_{B_{p}(X)} \approx\left(\sum_{n=0}^{\infty}\left\|x_{n}\right\|^{p} 2^{-n}\right)^{1 / p} .
$$

Let us mention some estimates which hold true in the scalar-valued case. For $p=2$, from Plancherel's theorem, we have

$$
\|f\|_{B_{2}} \approx\left(\sum_{n=0}^{\infty} \frac{\left|\alpha_{n}\right|^{2}}{n+1}\right)^{1 / 2}
$$

For each $1 \leq p \leq 2$ there exist positive constants $C_{p}$ and $C_{p}^{\prime}$ such that

$$
\left(\sum_{n=1}^{\infty} \frac{\left|\alpha_{n}\right|^{p}}{n^{3-p}}\right)^{1 / p} \leq C_{p}\|f\|_{B_{p}}
$$

and

$$
\left\|\left(\frac{\alpha_{n}}{n^{1 / p}}\right)\right\|_{p^{\prime}, p} \leq C_{p}^{\prime}\|f\|_{B_{p}}
$$

for all $f(z)=\sum_{n=0}^{\infty} \alpha_{n} z^{n} \in B_{p}$.

For each $2 \leq q<\infty$ there exists a positive constant $C_{q}^{\prime \prime}$ such that

$$
\|f\|_{B_{q}} \leq C_{q}^{\prime \prime}\left\|\left(\frac{\alpha_{n}}{n^{1 / q}}\right)\right\|_{q^{\prime}, q}
$$

for all $\left(x_{n}\right) \in \ell\left(q^{\prime}, q, X\right)$ and where $f(z)=\sum_{n=0}^{\infty} \alpha_{n} z^{n}$.

These results follow easily by interpolation and duality. Proposition 3.1 gives (9), Theorem 3.2 gives (10) and a duality argument gives (11).

Let us study the validity of these inequalities in the vector valued setting.

Remark 3.1 Let us point out that (9) actually follows from (10). 
Indeed, for $1 \leq p \leq 2$,

$$
\begin{aligned}
\sum_{n=1}^{\infty} \frac{\left\|x_{n}\right\|^{p}}{n^{3-p}} & \leq C \sum_{k=0}^{\infty}\left(\sum_{n \in I_{k}}\left\|x_{n}\right\|^{p}\right) 2^{-k(3-p)} \\
& \leq C \sum_{k=0}^{\infty}\left(\sum_{n \in I_{k}}\left\|x_{n}\right\|^{p^{\prime}}\right)^{p / p^{\prime}} 2^{k\left(1-p / p^{\prime}\right)} 2^{-k(3-p)} \\
& =C \sum_{k=0}^{\infty}\left(\sum_{n \in I_{k}}\left\|x_{n}\right\|^{p^{\prime}}\right)^{p / p^{\prime}} 2^{-k} \\
& \approx\left\|\left(\frac{x_{n}}{n^{1 / p}}\right)\right\|_{p^{\prime}, p}^{p} .
\end{aligned}
$$

Remark 3.2 Let $1 \leq p, q<\infty, \alpha \in \mathbb{R}, X=\ell_{q}$ and $F(z)=\sum_{n=1}^{\infty} x_{n} z^{n}$ with $x_{n}=n^{\alpha} e_{n}$ where $\left(e_{n}\right)$ stands for the canonical basis of $\ell_{q}$.

A simple computation shows that

$$
\left(\frac{x_{n}}{n^{1 / p}}\right) \in \ell\left(p^{\prime}, p, \ell_{q}\right) \text { if and only if } \alpha<1 / p-1 / p^{\prime}
$$

and

$$
F \in B_{p}\left(\ell_{q}\right) \text { if and only if } \alpha<1 / p-1 / q .
$$

The following example shows that the estimates (9) (and hence (10)) and (11) do not hold in general for vector-valued functions.

Example 3.1 Let $1<p \leq 2 \leq q<\infty$. Then $X=\ell_{r}$ fails (9) (and hence (10)) for any $r>p^{\prime}$ and $X=\ell_{s}$ fails (11) for any $s<q^{\prime}$.

PROOF. Assume $1<p \leq 2$ and take $\alpha=1 / p-1 / p^{\prime}$. Hence (13) implies that $F \in B_{p}\left(\ell_{r}\right)$. On the other hand $\left\|x_{n}\right\|=n^{\alpha}$ and then $\sum_{n=1}^{\infty} \frac{\left\|x_{n}\right\|^{p}}{n^{3-p}}=$ $\sum_{n=1}^{\infty} \frac{1}{n}=\infty$.

Assume $2 \leq q<\infty, s<q^{\prime}$ and take $\alpha=1 / q-1 / s$. Now (12) and (13) give that $\left(\frac{x_{n}}{n^{1 / q}}\right) \in \ell\left(q^{\prime}, q, \ell_{s}\right)$ but $F \notin B_{q}\left(\ell_{s}\right)$.

This leads to the following definitions: 
De fitition 3.4 Let $X$ be a complex Banach space and $1 \leq a \leq 2 \leq b<\infty$. $X$ is said to have Bergman type a (respect. Bergman cotype $b$ ) if there exists a constant $C_{a}>0$ (respect. $C_{b}>0$ ) such that

$$
\left.\left\|\left(\frac{x_{n}}{n^{1 / a}}\right)\right\|\right|_{a^{\prime}, a} \leq C_{a}\|f\|_{B_{a}(X)}
$$

for all $f(z)=\sum_{n=0}^{\infty} x_{n} z^{n} \in B_{a}(X)$,

(respect.

$$
\|f\|_{B_{b}(X)} \leq C_{b}\left\|\left(\frac{x_{n}}{n^{1 / b}}\right)\right\|_{b^{\prime}, b}
$$

for all $\left(x_{n}\right) \in \ell\left(b^{\prime}, b, X\right)$ where $f(z)=\sum_{n=0}^{\infty} x_{n} z^{n}$.)

Remark 3.3 Any Banach space has Bergman type 1 (see Theorem 3.2).

There is also a notion for $q=\infty$ in the previous definition. We need to consider the extreme case as

$$
\operatorname{Bloch}(X)=\left\{f: \mathbb{D} \rightarrow X \text { analytic }: \sup _{|z|<1}\left(1-|z|^{2}\right)|| f^{\prime}(z) \|<\infty\right\},
$$

and to observe that

$$
\sup _{|z|<1}\left(1-|z|^{2}\right)|| f^{\prime}(z)\|\leq C\| \leq\left\|\left(x_{n}\right)\right\|_{1, \infty} .
$$

Let us show that they are dual notions.

Proposition 3.5 Let $1<a \leq 2$ and $X$ be a Banach space. Then $X$ has Bergman type $a$ if and only if $X^{*}$ has Bergman cotype $a^{\prime}$.

PROOF. Note first that

$$
\sum_{n=0}^{\infty} \frac{\left\langle x_{n}^{*}, x_{n}\right\rangle}{n+1}=\int_{\mathbb{D}}\langle f(z), g(\bar{z})\rangle d A(z),
$$

for any $g(z)=\sum_{n=0}^{\infty} x_{n}^{*} z^{n}$ and $f(z)=\sum_{n=0}^{\infty} x_{n} z^{n}$.

Let us assume $X$ has Bergman type $a$, take $\left(x_{n}^{*}\right) \in \ell\left(a, a^{\prime}, X^{*}\right)$ and define $f(z)=\sum_{n=0}^{\infty} x_{n}^{*} z^{n}$. 
Then, for any $g(z)=\sum_{n=0}^{\infty} x_{n} z^{n} \in B_{a}(X)$, using (16) one gets

$$
\begin{aligned}
\left|\int_{\mathbb{D}}\langle f(z), g(\bar{z})\rangle d A(z)\right| & \leq\left\|\left(\frac{x_{n}}{(n+1)^{1 / a^{\prime}}}\right)\right\|_{a^{\prime}, a}\left\|\left(\frac{x_{n}^{*}}{(n+1)^{1 / a}}\right)\right\|_{a, a^{\prime}} \\
& \leq C\|g\|_{B_{a}(X)}\left\|\left(\frac{x_{n}^{*}}{n^{1 / a}}\right)\right\|_{a, a^{\prime}} .
\end{aligned}
$$

By the duality $\left(B_{a}(X)\right)^{*}=B_{a^{\prime}}\left(X^{*}\right)$ (see [5]) one has

$$
\|f\|_{B_{a^{\prime}}\left(X^{*}\right)}=\sup \left\{\left|\int_{\mathbb{D}}\langle f(z), g(\bar{z})\rangle d A(z)\right|:\|g\|_{B_{a}(X)}=1\right\} .
$$

Therefore $\|f\|_{B_{a^{\prime}}\left(X^{*}\right)} \leq C\left\|\left(\frac{x_{n}^{*}}{n^{1 / a}}\right)\right\|_{a, a^{\prime}}$.

Let us assume $X^{*}$ has Bergman cotype $a^{\prime}$ and take $f(z)=\sum_{n=0}^{\infty} x_{n} z^{n}$.

For any $\left(x_{n}^{*}\right)_{n}$ such that $\left\|\left(x_{n}^{*}\right)\right\|_{a, a^{\prime}}=1$, using (16), we have

$$
\begin{aligned}
\left|\sum_{n=0}^{\infty}\left\langle n^{-1 / a} x_{n}, x_{n}^{*}\right\rangle\right| & \leq\|f\|_{B_{a}(X)}\left\|\sum_{n=0}^{\infty}(n+1) n^{-1 / a} x_{n} z^{n}\right\|_{B_{a^{\prime}}\left(X^{*}\right)} \\
& \leq C\|f\|_{B_{a}(X)}\left\|\left(\frac{n+1}{n} x_{n}^{*}\right)\right\|_{a, a^{\prime}} \\
& \leq C\|f\|_{B_{a}(X)} .
\end{aligned}
$$

Now use

$$
\left\|\left(\frac{x_{n}}{n^{1 / a}}\right)\right\|_{a^{\prime}, a}=\sup \left\{\left|\sum_{n=0}^{\infty}\left\langle n^{-1 / a} x_{n}, x_{n}^{*}\right\rangle\right|:\left\|\left(x_{n}^{*}\right)\right\|_{a, a^{\prime}}=1\right\},
$$

to get that $X$ has Bergman type $a$.

Let us recall a well known notion which is very much related to the previous ones.

Let $1 \leq p \leq 2$. A Banach space $X$ is said to have Fourier type $p$ if there exists a constant $\mathrm{C}$ such that

$$
\left(\sum_{n=-\infty}^{\infty}\|\hat{f}(n)\|^{p^{\prime}}\right)^{1 / p^{\prime}} \leq C\|f\|_{L^{p}(X)}
$$

for all function $f \in L^{p}(\mathbb{T}, X)$.

It was first introduced by J. Peetre (see [29]). We refer the reader to the survey [20] for a complete study and references about this property. 
We just point out here the equivalent formulation:

There exists a constant $C>0$ such that for all $\left(x_{n}\right) \in \ell_{p}(X)$ the function $f(t)=\sum_{n=-\infty}^{\infty} x_{n} e^{i n t}$ belongs to $L^{p^{\prime}}(\mathbb{T}, X)$ and

$$
\|f\|_{L^{p^{\prime}(X)}} \leq C\left\|\left(x_{n}\right)\right\|_{p} .
$$

It is not difficult to see that $X$ has Fourier type $p$ if and only if $X^{*}$ does have it. The main examples are $L^{r}(\mu)$ for any $p \leq r \leq p^{\prime}$ or interpolation spaces between any Banach space $X_{0}$ and any Hilbert space $X_{1},\left[X_{0}, X_{1}\right]_{\theta}$ where $1 / p=1-\theta / 2$.

Theorem 3.6 Let $1<p \leq 2$. If $X$ has Fourier type $p$ then $X$ has Bergman type $p$ and Bergman cotype $p^{\prime}$.

PROOF. Using Lemma 2.6 we have

$$
\sum_{k=1}^{\infty}\left(\sum_{n \in I_{k}} \frac{\left\|x_{n}\right\|^{p^{\prime}}}{n^{p^{\prime} / p}}\right)^{p / p^{\prime}} \approx \int_{0}^{1}\left(\sum_{n=1}^{\infty}\left\|x_{n}\right\|^{p^{\prime}} r^{n p^{\prime}}\right)^{p / p^{\prime}} d r .
$$

Since $X$ has Fourier type $p$

$$
\left(\sum_{n=1}^{\infty}\left\|x_{n}\right\|^{p^{\prime}} r^{p^{\prime} n}\right)^{1 / p^{\prime}} \leq C M_{p}(f, r)
$$

which implies that

$$
\left\|\left(\frac{x_{n}}{n^{1 / p}}\right)\right\|_{p^{\prime}, p}^{p} \leq C \int_{0}^{1} M_{p}^{p}(f, r) d r
$$

To get that $X$ has Bergman cotype $p^{\prime}$, one can use either the dual formulation with Bergman type of $X^{*}$ or repeat the previous argument using now $M_{p^{\prime}}(f, r) \leq C\left(\sum_{n=1}^{\infty}\left\|x_{n}\right\|^{p} r^{n p}\right)^{1 / p}$.

Corollary 3.7 Let $1 \leq p \leq 2$. Then $L^{r}(\mu)$ has Bergman type $p$ and cotype $p^{\prime}$ for $p \leq r \leq p^{\prime}$.

Let us state a little extension of Theorem 3.6 that we used later on. 
Theorem 3.8 Let $1<a \leq 2$ and let $X$ be a Banach space with Fourier type $a$. If $p \geq a$ then there exists a constant $C>0$ such that

$$
\left\|\left(\frac{x_{n}}{n^{1 / p}}\right)\right\|_{a^{\prime}, p} \leq C\|f\|_{B_{p}(X)}
$$

for all $f(z)=\sum_{n=1}^{\infty} x_{n} z^{n}$.

If $p \leq a^{\prime}$ then there exists a constant $C>0$ such that

$$
\|f\|_{B_{p}(X)} \leq C\left\|\left(\frac{x_{n}}{n^{1 / p}}\right)\right\|_{a, p}
$$

for all $\left(\frac{x_{n}}{n^{1 / p}}\right) \in \ell(a, p, X)$ and $f(z)=\sum_{n=1}^{\infty} x_{n} z^{n}$.

PROOF. We need only to use that $\left(\int_{0}^{1} M_{a}^{p}(f, r) d r\right)^{1 / p} \leq C\|f\|_{B_{p}(X)}$ for $p \geq a$ or $\|f\|_{B_{p}(X)} \leq C\left(\int_{0}^{1} M_{a^{\prime}}^{p}(f, r) d r\right)^{1 / p}$ for $p \leq a^{\prime}$ and then use (17) or (18) respectively, together with Lemma 2.6 to get the result.

We shall first show an extension to the vector valued setting of one inequality due to Hardy and Littlewood (see [16, 23]). Our proof is a modification of the one given in Theorem 2.1 of [14] for the Hardy space $H^{1}(X)$, but we include it here for the sake of completeness.

Theorem 3.9 Let $X$ be a Banach space and let $1 \leq p<q<\infty$. Then

$$
\left(\int_{0}^{1} \int_{0}^{1}(1-r)^{-p / q} M_{q}^{p}(f, r s) d r d s\right)^{1 / p} \leq C\|f\|_{B_{p}(X)} .
$$

PROOF. We shall actually prove that

$$
\left(\int_{0}^{1}(1-r)^{-p / q} M_{q}^{p}(f, r) d r\right)^{1 / p} \leq C\|f\|_{H_{p}(X)},
$$

where $H_{p}(X)$ stands for the vector-valued Hardy space.

Let us first recall that if $0<p \leq q \leq \infty$ and $g$ is an $X$-valued analytic function then, using that $g\left(r^{2} e^{i t}\right)=P_{r} * g\left(r e^{i t}\right)$, one gets

$$
M_{q}\left(g, r^{2}\right) \leq C(1-r)^{1 / q-1 / p} M_{p}(g, r)
$$


Now let us take $0<p_{1}<p<p_{2}<q$, and apply (19) to get for $i=1,2$

$$
(1-r)^{-1 / q} M_{q}(f, r) \leq C(1-r)^{-1 / p_{i}}\|f\|_{H^{p_{i}(X)}} .
$$

Hence

$$
\left|\left\{r \in[0,1]:(1-r)^{-1 / q} M_{q}(f, r)>\lambda\right\}\right| \leq C \frac{\|f\|_{H^{p_{i}(X)}}^{p_{i}}}{\lambda^{p_{i}}} .
$$

This actually gives that

$$
f \rightarrow F\left(r, e^{i t}\right)=(1-r)^{-1 / q} f\left(r e^{i t}\right)
$$

defines a bounded operator from $H^{p_{i}}(X)$ into the vector valued Lorenz space $L^{p_{i}, \infty}\left(d r, L^{q}(X)\right)$.

Now using the standard real method of interpolation for $\theta \in(0,1)$ such that $(1-\theta) / p_{1}+\theta / p_{2}=1 / p$ we have (see [4])

$$
\left(L^{p_{1}, \infty}\left(d r, L^{q}(X)\right), L^{p_{2}, \infty}\left(d r, L^{q}(X)\right)\right)_{\theta, p}=L^{p}\left(d r, L^{q}(X)\right) .
$$

On the other hand, since the Banach space $X$ is the same for both indices, it is not difficult to extend the scalar-valued proof (see [15] and references there) to get

$$
\left(H^{p_{1}}(X), H^{p_{2}}(X)\right)_{\theta, p}=H^{p}(X) .
$$

Hence the operator is bounded from $H^{p}(X)$ to $L^{p}\left(d r, L^{q}(X)\right)$, that is

$$
\int_{0}^{1}(1-r)^{-p / q} M_{q}^{p}(f, r) d r \leq C\|f\|_{H^{p}(X)}^{p} .
$$

Now the proof is finished by integrating over $(0,1)$.

An application of Theorem 3.9 allows us to improve Theorems 3.6 and 3.8 .

Theorem 3.10 Let $1<a \leq 2$ and let $X$ be a Banach space with Fourier type a.

If $p<a$ and $\alpha=1 / p-1 / a$ then there exists a constant $C>0$ such that

$$
\left\|\left(\frac{x_{n}}{n^{\alpha+1 / p}}\right)\right\|_{a^{\prime}, p} \leq C\|f\|_{B_{p}(X)}
$$

for all $f(z)=\sum_{n=1}^{\infty} x_{n} z^{n}$. 
PROOF. Since $X$ has Fourier type $a$, we have

$$
\left(\sum_{n=1}^{\infty}\left\|x_{n}\right\|^{a^{\prime}} r^{p^{\prime} n}\right)^{1 / a^{\prime}} \leq C M_{a}(f, r),
$$

Using Theorem 3.9 and Lemma 2.6 we get

$$
\begin{aligned}
\|f\|_{B_{p}(X)} & \geq C\left(\int_{0}^{1} \int_{0}^{1}(1-r)^{-p / a} M_{a}^{p}(f, r s) d r d s\right)^{1 / p} \\
& \geq C\left(\int_{0}^{1} \int_{0}^{1}(1-r)^{-p / a}\left(\sum_{n=1}^{\infty}\left\|x_{n}\right\|^{a^{\prime}} s^{a^{\prime} n} r^{a^{\prime} n}\right)^{p / a^{\prime}} d r d s\right)^{1 / p} \\
& \geq C\left(\int_{0}^{1}(1-r)^{-p / a}\left(\int_{0}^{1}\left(\sum_{n=1}^{\infty}\left\|x_{n}\right\|^{a^{\prime}} s^{a^{\prime} n} r^{a^{\prime} n}\right)^{p / a^{\prime}} d s\right) d r\right)^{1 / p} \\
& \geq C\left(\int_{0}^{1}(1-r)^{-p / a} \sum_{k=1}^{\infty}\left(\sum_{n \in I_{k}} \frac{\left\|r^{n} x_{n}\right\|^{a^{\prime}}}{n^{a^{\prime} / p}}\right)^{p / a^{\prime}} d r\right)^{1 / p} \\
& \geq C\left(\int_{0}^{1}(1-r)^{-p / a} \sum_{k=1}^{\infty}\left(\sum_{n \in I_{k}}\left\|x_{n}\right\|^{a^{\prime}}\right)^{p / a^{\prime}} r^{p 2^{k}} 2^{-k} d r\right)^{1 / p} \\
& \geq C\left(\sum_{k=1}^{\infty}\left(\sum_{n \in I_{k}}\left\|x_{n}\right\|^{a^{\prime}}\right)^{p / a^{\prime}}\left(\int_{0}^{1}(1-r)^{-p / a} r^{p 2^{k}} 2^{-k} d r\right)\right)^{1 / p} \\
& \left.\geq C\left(\sum_{k=1}^{\infty}\left(\sum_{n \in I_{k}}\left\|x_{n}\right\|^{a^{\prime}}\right)^{p / a^{\prime}} 2^{-k(1-p / a)} 2^{-k} d r\right)\right)^{1 / p} \\
& \geq C\left\|\left(\frac{x_{n}}{n^{\alpha+1 / p}}\right)\right\|_{a^{\prime}, p}
\end{aligned}
$$

\section{Multipliers from $B_{p}(X)$ to $\ell_{q}(Y)$.}

In this section we analyze the vector-valued versions of (3) and (4).

Theorem 4.1 Let $X$ and $Y$ two complex Banach spaces, and let $1 \leq q \leq \infty$. A sequence $\left(T_{n}\right) \in\left(B_{1}(X), \ell_{q}(Y)\right)$ if and only if the sequence $\left(n T_{n}\right)$ defines a bounded operator from $X$ to $\ell(q, \infty, Y)$. 
PROOF. Assume that $\left(T_{n}\right) \in\left(B_{1}(X), \ell_{q}(Y)\right)$. Hence one has that $\left(T_{n}(x)\right) \in$ $\left(B_{1}, \ell_{q}(Y)\right)$ for all $x \in X$. Now for each $z \in \mathbb{D}$ we define $K_{z}(w)=\frac{1}{(1-w z)^{3}}$. Since $\left\|K_{z}\right\|_{B_{1}} \approx \frac{1}{1-|z|}$, the assumption implies that

$$
\left(\sum_{n=0}^{\infty} n^{2 q}|| T_{n}(x)||^{q}|z|^{n q}\right)^{1 / q} \leq \frac{C}{1-|z|}
$$

for each $z \in \mathbb{D}$ and $\|x\| \leq 1$. Invoking now Lemma 2.4 for $q<\infty$ one gets that $\left(n^{q}\left\|T_{n}(x)\right\|^{q}\right) \in \ell(1, \infty, Y)$, or equivalently that $\left(n\left\|T_{n}(x)\right\|\right) \in$ $\ell(q, \infty, Y)$, and that $x \rightarrow\left(n T_{n}(x)\right)$ is a bounded operator from $X$ into $\ell(q, \infty, Y)$. The case $q=\infty$ follows from Lemma 2.5.

Let us assume now, for $q<\infty$, that

$$
\sup _{\|x\| \leq 1} \sup _{k \geq 0} \sum_{n \in I_{k}} n^{q}\left\|T_{n}(x)\right\|^{q}=A^{q}<\infty
$$

Then, if $f(z)=\sum_{n=0}^{\infty} x_{n} z^{n}$, using Theorem 3.2 one gets

$$
\begin{aligned}
\sum_{n=0}^{\infty}\left\|T_{n}\left(x_{n}\right)\right\|^{q} & =\sum_{k=0}^{\infty} \sum_{n \in I_{k}}\left\|T_{n}\left(\frac{x_{n}}{\left\|x_{n}\right\|}\right)\right\|^{q}\left\|x_{n}\right\|^{q} \\
& \leq \sum_{k=0}^{\infty}\left(\sum_{n \in I_{k}} n^{q}\left\|T_{n}\left(\frac{x_{n}}{\left\|x_{n}\right\|}\right)\right\|^{q}\right) \sup _{n \in I_{k}} \frac{\left\|x_{n}\right\|^{q}}{n^{q}} \\
& \leq \sup _{k \geq 0} \sum_{n \in I_{k}} n^{q}\left\|T_{n}\left(\frac{x_{n}}{\left\|x_{n}\right\|}\right)\right\|^{q} \sum_{k=0}^{\infty}\left(\sup _{n \in I_{k}} \frac{\left\|x_{n}\right\|^{q}}{n^{q}}\right) \\
& \leq A^{q}\left(\sum_{k=0}^{\infty} \sup _{n \in I_{k}} \frac{\left\|x_{n}\right\|}{n}\right)^{q} \\
& \leq A^{q}\|f\|_{B_{1}(X)}^{q}
\end{aligned}
$$

The case $q=\infty$ is immediate from Proposition 3.1.

Corollary 4.2 Let $X$ a Banach space and let $\left(x_{n}^{*}\right)$ be a sequence in $X^{*}$ such that

$$
\sum_{n=0}^{\infty}\left|\left\langle x_{n}^{*}, x_{n}\right\rangle\right| \leq C \int_{D}\|f(z)\| d A(z)
$$


for all $f(z)=\sum_{n=0}^{\infty} x_{n} z^{n}$. Then there exists a constant $C>0$ such that

$$
\sup _{\|x\|=1} \sum_{n \in I_{k}}\left|\left\langle x_{n}^{*}, x\right\rangle\right| \leq C 2^{-k}
$$

for all $k \in \mathbb{N}$ and $x \in X$.

Let us point out some necessary and some sufficient conditons for $\left(T_{n}\right)$ to belong to $\left(B_{p}(X), \ell_{q}(Y)\right)$ for $1<p<\infty$.

Proposition 4.3 Let $1 \leq p<\infty, 1 \leq q \leq \infty$ and $1 / r=((1 / q)-(1 / p))^{+}$. Then

$$
\left(B_{p}(X), \ell_{q}(Y)\right) \subset\left\{\left(T_{n}\right) \in L(X, Y):\left\|T_{n}\right\|=O\left(n^{-1 / p}\right)\right\}
$$

and

$$
\left\{\left(T_{n}\right):\left(n^{2 / p}\left\|T_{n}\right\|\right) \in \ell_{r}\right\} \subset\left(B_{p}(X), \ell_{q}(Y)\right) .
$$

PROOF. If we set $u_{n}(z)=z^{n}$, then $\left\|u_{n} \otimes x\right\|_{B_{p}(X)} \sim n^{-1 / p}\|x\|$.

Now if $\left(T_{n}\right) \in\left(B_{p}(X), \ell_{q}(Y)\right)$ then $\left\|T_{n} x\right\| \leq C n^{-1 / p}\|x\|$ for any $x \in X$, so that $\left\|T_{n}\right\| \leq C n^{-1 / p}$.

For the second embedding, Propositon 3.1 gives that

$$
\left\|\left(T_{n}\left(x_{n}\right)\right)\right\|_{q} \leq\left\|\left(n^{2 / p}\left\|T_{n}\right\|\right)\right\|_{r}\left\|\left(\frac{\left\|x_{n}\right\|}{n^{2 / p}}\right)\right\|_{p} \leq C\|f\|_{B_{p}(X)} .
$$

A direct application of Theorems 3.2 and 2.3 produce better information on multipliers.

Proposition 4.4 Let $1 \leq p<\infty, 1 \leq q \leq \infty$ and let $X, Y$ be Banach spaces then

$$
\left(B_{p}(X), \ell_{q}(Y)\right) \subset\left\{\left(T_{n}\right):\left(n^{1 / p} T_{n}\right) \in \ell(\infty, r, \mathcal{L}(X, Y))\right\}
$$

and

$$
\left\{\left(T_{n}\right):\left(n^{1 / p} T_{n}\right) \in \ell(q, r, \mathcal{L}(X, Y))\right\} \subset\left(B_{p}(X), \ell_{q}(Y)\right)
$$

where $1 / r=((1 / q)-(1 / p))^{+}$.

Let us show how to improve the previous propositions under certain assumptions on $X$. 
Theorem 4.5 Let $1 \leq p<\infty, 1 \leq q \leq \infty, 1 \leq a \leq 2$ and let $X$ be $a$ complex Banach space of Fourier type $a$. Put $1 / s=\left((1 / q)-\left(1 / a^{\prime}\right)\right)^{+}, 1 / t=$ $((1 / q)-(1 / a))^{+}$and $1 / r=((1 / q)-(1 / p))^{+}$.

If $1 \leq p<a$ and $\alpha=1 / p-1 / a$ then

$$
\left\{\left(T_{n}\right):\left(n^{\alpha+1 / p} T_{n}\right) \in \ell(s, r, \mathcal{L}(X, Y))\right\} \subset\left(B_{p}(X), \ell_{q}(Y)\right) .
$$

If $a \leq p$ then

$$
\left\{\left(T_{n}\right):\left(n^{1 / p} T_{n}\right) \in \ell(s, r, \mathcal{L}(X, Y))\right\} \subset\left(B_{p}(X), \ell_{q}(Y)\right) .
$$

PROOF. By Theorem 2.3, $\ell(s, r, \mathcal{L}(X, Y))=\left(\ell\left(a^{\prime}, p, X\right), \ell_{q}(Y)\right)$. Let us assume that

$$
\left(n^{\alpha+1 / p} T_{n}\right) \in\left(\ell\left(a^{\prime}, p, X\right), \ell_{q}(Y)\right) \text { or }\left(n^{1 / p} T_{n}\right) \in\left(\ell\left(a^{\prime}, p, X\right), \ell_{q}(Y)\right) .
$$

for $1 \leq p \leq a$ or $a \leq p$ respectively where $\alpha=1 / p-1 / a$.

Now Theorem 3.10 gives, for $1 \leq p<a$, that

$$
\left\|\left(T_{n} x_{n}\right)\right\|_{q}=\left\|\left(n^{\alpha+1 / p} T_{n}\left(\frac{x_{n}}{n^{1 / p+\alpha}}\right)\right)\right\|_{q} \leq C\left\|\left(n^{-1 / p-\alpha} x_{n}\right)\right\|_{a^{\prime}, p} \leq C\|f\|_{B_{p}(X)} .
$$

and Theorem 3.8 implies, for $a \leq p$, that

$$
\left\|\left(T_{n} x_{n}\right)\right\|_{q}=\left\|\left(n^{1 / p} T_{n}\left(\frac{x_{n}}{n^{1 / p}}\right)\right)\right\|_{q} \leq C\left\|\left(n^{-1 / p} x_{n}\right)\right\|_{a^{\prime}, p} \leq C\|f\|_{B_{p}(X)} .
$$

These two estimates give (20) and (21).

Theorem 4.6 Let $1 \leq p \leq 2$ and let $X$ be a complex Banach space. The following statements are equivalent:

(i) $X$ has Bergman type $p$.

(ii) For any $1 \leq q<\infty$ and for any other Banach space $Y$,

$$
\left\{\left(T_{n}\right):\left(n^{1 / p} T_{n}\right) \in \ell(s, r, \mathcal{L}(X, Y))\right\} \subset\left(B_{p}(X), \ell_{q}(Y)\right)
$$

where $1 / s=\left((1 / q)-\left(1 / p^{\prime}\right)\right)^{+}$y $1 / r=((1 / q)-(1 / p))^{+}$.

PROOF. Let us assume $X$ has Bergman type $p$. Now (ii) follows from the embedding $B_{p}(X) \subset\left\{\left(x_{n}\right):\left(n^{-1 / p} x_{n}\right) \in \ell\left(p^{\prime}, p, X\right)\right\}$. 
Assume (ii) for $Y=\mathbb{C}$ and $q=1$. Then any $\left(x_{n}^{*}\right)$ such that $\left\|\left(n^{1 / p} x_{n}^{*}\right)\right\|_{p, p^{\prime}}<$ $\infty$ gives a multiplier in $\left(B_{p}(X), \ell_{1}\right)$. Therefore there exists $C>0$ such that

$$
\sum_{n=0}^{\infty}\left|\left\langle x_{n}^{*}, x_{n}\right\rangle\right| \leq C\|f\|_{B_{p}(X)}\left\|\left(n^{1 / p} x_{n}^{*}\right)\right\|_{p, p^{\prime}}
$$

for any $f(z)=\sum_{n=0}^{\infty} x_{n} z^{n}$.

¿From duality now one gets $\left\|\left(n^{-1 / p} x_{n}\right)\right\|_{p^{\prime}, p} \leq C\|f\|_{B_{p}(X)}$.

Theorem 4.7 Let $2 \leq p<\infty$ and let $X$ be a complex Banach space of Bergman cotype $p$. Then for any $1 \leq q<\infty$ and for any other Banach space $Y$,

$$
\left(B_{p}(X), \ell_{q}(Y)\right) \subset\left\{\left(T_{n}\right):\left(n^{1 / p} T_{n}\right) \in \ell(s, r, \mathcal{L}(X, Y))\right\}
$$

where $1 / s=\left((1 / q)-\left(1 / p^{\prime}\right)\right)^{+}$y $1 / r=((1 / q)-(1 / p))^{+}$.

PROOF. The assumption means that $\left\{\left(x_{n}\right):\left(\frac{x_{n}}{n^{1 / p}}\right) \in \ell\left(p^{\prime}, p, X\right)\right\} \subset B_{p}(X)$. Therefore $\left(T_{n}\right) \in\left(B_{p}(X), \ell_{q}(Y)\right)$ gives $\left(n^{1 / p} T_{n}\right) \in\left(\ell\left(p^{\prime}, p, X\right), \ell_{q}(Y)\right)$. Now using Theorem 2.3 the proof is finished.

Recall the well known notion of Rademacher type (see [28]).

For $1 \leq p \leq 2$ a Banach space $X$ is said to have Rademacher type $p$ if there exists a constant $C$ such that

$$
\int_{0}^{1}\left\|\sum_{j=1}^{n} x_{j} r_{j}(t)\right\| d t \leq C\left(\sum_{j=1}^{n}\left\|x_{j}\right\|^{p}\right)^{1 / p}
$$

for any finite family $x_{1}, x_{2}, \ldots x_{n}$ of vectors in $X$ where $r_{j}$ stand for the Rademacher functions on $[0,1]$.

It is known and easy to see that Fourier type $p$ implies Rademacher type $p$.

Theorem 4.8 Let $1 \leq p<\infty, 1 \leq q \leq \infty, 1 \leq a \leq 2$, and let $X$ be $a$ complex Banach space of Rademacher type $a$ and $Y$ be any Banach space. Then

$$
\left(B_{p}(X), \ell_{q}(Y)\right) \subset\left\{\left(T_{n}\right):\left(n^{1 / p} T_{n}\right) \in \ell(s, r, \mathcal{L}(X, Y))\right\},
$$

where $1 / s=((1 / q)-(1 / a))^{+}$and $1 / r=((1 / q)-(1 / p))^{+}$. 
PROOF. Let $\widetilde{T}: B_{p}(X) \rightarrow \ell_{q}(Y)$ the bounded linear operator defined by $\left(T_{n}\right)$ as a multiplier, i.e. $\widetilde{T} f=\left(T_{n} x_{n}\right)$ for every analytic polynomial $f(z)=$ $\sum_{n>0} x_{n} z^{n}$.

For any $t \in[0,1]$, let $f_{t}$ the polynomial given by $f_{t}(z)=\sum_{n \geq 0} r_{n}(t) x_{n} z^{n}$, where $\left(r_{n}\right)$ is the sequence of Rademacher functions.

It's clear that $\left\|\widetilde{T} f_{t}\right\|=\|\widetilde{T} f\|$ for every $t$, and then

$$
\begin{aligned}
\left\|\left(T_{n} x_{n}\right)\right\|_{q}^{p} & =\|\widetilde{T} f\|_{q}^{p}=\int_{0}^{1}\left\|\widetilde{T} f_{t}\right\|^{p} d t \leq\|\widetilde{T}\|^{p} \int_{0}^{1}\left\|f_{t}\right\|_{B_{p}(X)}^{p} d t \\
& =\frac{\|\widetilde{T}\|^{p}}{2 \pi} \int_{0}^{1} \int_{-\pi}^{\pi} \int_{0}^{1}\left\|\sum_{n \geq 0} r_{n}(t) x_{n} r^{n} e^{i n \theta}\right\|^{p} d t d \theta d r .
\end{aligned}
$$

Since $X$ is of type $a$, we have for every $\theta$ that

$$
\int_{0}^{1}\left\|\sum_{n \geq 0} r_{n}(t) x_{n} r^{n} e^{i n \theta}\right\|^{p} d t \leq C\left(\sum_{n \geq 0}\left\|x_{n}\right\|^{a} r^{n a}\right)^{p / a}
$$

and integrating this in $[-\pi, \pi]$ we get

$$
\left\|\left(T_{n} x_{n}\right)\right\|_{q}^{p} \leq C\|\widetilde{T}\|^{p} \int_{0}^{1}\left(\sum_{n \geq 0}\left\|x_{n}\right\|^{a} r^{n a}\right)^{p / a} d r .
$$

Now Lemma 2.6 yields

$$
\left\|\left(T_{n} x_{n}\right)\right\|_{q}^{p} \leq C \int_{0}^{1}\left(\sum_{n \geq 0}\left\|x_{n}\right\|^{a} r^{n}\right)^{p / a} d r \leq C \sum_{k}\left(\sum_{n \in I_{k}} \frac{\left\|x_{n}\right\|^{a}}{n^{a / p}}\right)^{p / a},
$$

which gives $\left\|\left(T_{n} x_{n}\right)\right\|_{q} \leq C\left\|\left(\frac{x_{n}}{n^{1 / p}}\right)\right\|_{\ell(a, p, X)}$.

We have thus shown that $\left(n^{1 / p} T_{n}\right) \in(\ell(a, p, X), \ell(q, q, Y))$, and by Theorem 2.3 this gives $\left(n^{1 / p} T_{n}\right) \in \ell(s, r, \mathcal{L}(X, Y))$.

Theorems 4.5 and 4.8 give a characterization of the multipliers from $B_{p}(X)$ to $\ell_{q}(Y)$ for $p \geq 2$ and $X$ being isomorphic to a Hilbert space.

Corollary 4.9 Let $1 \leq q \leq \infty$ and $2 \leq p<\infty$. If $X$ is isomorphic to a Hilbert space then

$$
\left.\left(B_{p}(X), \ell_{q}(Y)\right)=\left\{\left(T_{n}\right) \subset \mathcal{L}(X, Y) ;\left(n^{1 / p}\left\|T_{n}\right\|\right) \in \ell_{(} s, r\right)\right\},
$$

where $1 / s=((1 / q)-(1 / 2))^{+}$and $1 / r=((1 / q)-(1 / p))^{+}$.

We have, in particular, that $\left(T_{n}\right) \in\left(B_{2}(X), \ell_{q}(Y)\right)$ if and only if the sequence $\left(\sqrt{n}\left\|T_{n}\right\|\right) \in \ell_{s}$. 


\section{$5 \quad$ Multipliers from $B_{p}(X)$ to $B_{q}(Y)$.}

We now study the vector-valued versions of (6) and (5).

Theorem 5.1 Let $1 \leq a \leq 2 \leq b \leq \infty$ and let $X$ and $Y$ be complex Banach spaces, such that $X$ has Fourier type $a$ and $Y$ has Fourier type $b^{\prime}$. If $a \leq p<\infty$ and $1 \leq q \leq b$ then

$$
\left\{\left(T_{n}\right):\left(n^{-1 / r} T_{n}\right) \in \ell(\alpha, \beta, \mathcal{L}(X, Y))\right\} \subset\left(B_{p}(X), B_{q}(Y)\right)
$$

where $1 / r=(1 / q)-(1 / p), 1 / \alpha=(1 / a)-(1 / b)$ and $1 / \beta=(1 / r)^{+}$.

PROOF. By Theorem 2.3, the hypothesis says that

$$
\left(n^{-1 / r} T_{n}\right) \in \ell(\alpha, \beta, \mathcal{L}(X, Y))=\left(\ell\left(a^{\prime}, p, X\right), \ell\left(b^{\prime}, q, Y\right)\right) .
$$

Let $f$ be an analytic polynomial given by $f(z)=\sum_{n \geq 1} x_{n} z^{n}, x_{n} \in X$ and $g(z)=\sum_{n \geq 1} T_{n} x_{n} z^{n}$, which takes values in $Y$. Since $a \leq p$ and $q \leq b$, Theorem 3.8 implies

$$
\begin{aligned}
\|g\|_{B_{q}(Y)} & \leq C\left\|\left(\frac{T_{n} x_{n}}{n^{1 / q}}\right)\right\|_{b^{\prime}, q} \\
& \leq C\left\|\left(n^{(1 / p)-(1 / q)}\left\|T_{n}\right\| \frac{\left\|x_{n}\right\|}{n^{1 / p}}\right)\right\|_{b^{\prime}, q} \\
& \leq C\left\|\left(n^{-1 / r} T_{n}\right)\right\|_{\alpha, \beta}\left\|\left(\frac{x_{n}}{n^{1 / p}}\right)\right\|_{a^{\prime}, p} \\
& \leq C\|f\|_{B_{p}(X)} .
\end{aligned}
$$

Theorem 5.2 Let $1 \leq p, q<\infty$ and let $X$ and $Y$ be any complex Banach spaces. Then

$$
\left(B_{p}(X), B_{q}(Y)\right) \subset\left\{\left(T_{n}\right):\left(n^{-1 / r}\left\|T_{n}\right\|\right) \in \ell(\infty, s, \mathcal{L}(X, Y))\right\}
$$

where $1 / r=(1 / q)-(1 / p)$ and $1 / s=(1 / r)^{+}$.

PROOF. It follows from Theorem 3.2 that

$$
B_{q}(Y) \subset\left\{\left(y_{n}\right):\left(n^{-1 / q}\left\|y_{n}\right\|\right) \in \ell(\infty, q)\right\}
$$


and that

$$
\left\{\left(x_{n}\right):\left(n^{-1 / p}|| x_{n}||\right) \in \ell(1, p)\right\} \subset B_{p}(X) .
$$

Hence $\left(T_{n}\right) \in\left(B_{p}(X), B_{q}(Y)\right)$ implies $\left(n^{1 / p-1 / q} T_{n}\right) \in(\ell(1, p, X), \ell(\infty, q, Y))$ and the proof is finished.

Combining Theorems 5.1 and 5.2 we get the analogue to (6) for Hilbert valued functions.

Corollary 5.3 If $X$ and $Y$ are both of them isomorphic to complex Hilbert spaces and $1 \leq q \leq 2 \leq p<\infty$ then

$$
\left(B_{p}(X), B_{q}(Y)\right)=\left\{\left(T_{n}\right) ;\left(n^{-1 / r} T_{n}\right) \in \ell(\infty, r, \mathcal{L}(X, Y)\}\right.
$$

where $1 / r=(1 / q)-(1 / p)$.

Let us now state several lemmas that we shall need in the sequel to get the analogue to (5).

The next result can be carried over the vector-valued setting.

Lemma 5.4 (see [16], Theorem 5.5] ) Let $X$ be a complex Banach space, $f$ be an $X$-valued analytic function, $q \geq 1$ and $\alpha>0$. Then $M_{q}(f, r)=$ $O\left(\frac{1}{(1-r)^{\alpha}}\right)$ if and only if $M_{q}\left(f^{\prime}, r\right)=O\left(\frac{1}{(1-r)^{1+\alpha}}\right)$.

Lemma 5.5 (see [8], Lemma 2.1 ) Let $X$ be a complex Banach space and $f$ be an $X$-valued analytic function. Then $f \in B_{q}(X)$ if and only if

$$
\int_{0}^{1}(1-r)^{q} M_{q}^{q}\left(f^{\prime}, r\right) d r<\infty
$$

Lemma 5.6 (see [11], Lemma 5.2) Let $f$ be an $X$-valued analytic function and $q \in[1, \infty)$. Then the following are equivalent:

(i) $M_{q}(f, r)=O\left(\frac{1}{(1-r)^{1+1 / q}}\right)$.

(ii) $\left\|f_{s}\right\|_{B_{q}(X)}=O\left(\frac{1}{1-s}\right)$ where $f_{s}(z)=f(s z)$.

Let us now recall the definition of the Lipschitz classes in the vectorvalued setting. 
De fitition 5.7 Let $1 \leq p \leq \infty$ and $0<\alpha<1$. The Lipschizt class $\Lambda_{\alpha}^{p}(X)$ is formed by those functions $\phi \in L^{p}(\mathbb{T}, X)$ such that

$$
w_{p}(\phi, t)=\left(\int_{0}^{2 \pi}\left\|\phi\left(e^{i(t+s)}\right)-\phi\left(e^{i s}\right)\right\|^{p} \frac{d s}{2 \pi}\right)^{1 / p}=O\left(|t|^{\alpha}\right),(t \rightarrow 0) .
$$

Same proof as in the scalar valued case shows the following characterization of analytic Lipschitz functions.

Lemma 5.8 (see [16]) Let $1 \leq p \leq \infty, 0<\alpha<1$ and let $\phi \in L^{p}(\mathbb{T}, X)$ such that its Poisson extension $f$ is an $X$-valued analytic function. Then the following are equivalent:

(i) $M_{p}\left(f^{\prime}, r\right)=O\left(\frac{1}{(1-r)^{1-\alpha}}\right)$.

(ii) $\phi \in \Lambda_{\alpha}^{p}(X)$.

We shall use the following general result, that was used in [11] and [7] to get applications to multipliers, and whose vector-valued version was shown in more generality in [6].

Theorem 5.9 (see [6], Theorem 2.1) Let $X, Y$ be complex Banach spaces. Let $\Phi$ be a linear map from the $X$-valued polynomials into $Y$. Define $\Phi_{n}$ : $X \rightarrow Y$ by the formula $\Phi_{n}(x)=\Phi\left(x \otimes u_{n}\right)$ and $F_{\Phi}(z)=\sum_{n=0}^{\infty} \Phi_{n} z^{n}$. The following are equivalent:

(i) $\sup _{|z|<1}\left(1-|z|^{2}\right)|| F_{\Phi}^{\prime \prime}(z) \|_{\mathcal{L}(X, Y)}<\infty$

(ii) $\Phi$ extends to a bounded operator in $\mathcal{L}\left(B_{1}(X), Y\right)$.

Moreover $\|\Phi\| \approx\left\|F_{\Phi}(0)\right\|+\left\|F_{\Phi}^{\prime}(0)\right\|+\sup _{|z|<1}\left(1-|z|^{2}\right)\left\|F_{\Phi}^{\prime \prime}(z)\right\|$.

Theorem 5.10 Let $X$ and $Y$ be complex Banach spaces, a sequence of operators $\left(T_{n}\right) \subset \mathcal{L}(X, Y)$ and $1<q<\infty$. Then $\left(T_{n}\right) \in\left(B_{1}(X), B_{q}(Y)\right)$ if and only if the map $x \rightarrow F_{x}$ given by $F_{x}(z)=\sum_{n=1}^{\infty} T_{n}(x) z^{n}$ defines a bounded operator from $X$ into $\Lambda_{1 / q^{\prime}}^{q}(Y)$.

PROOF. If $\widetilde{T}: B_{1}(X) \longrightarrow B_{q}(Y)$ is the operator corresponding to $\left(T_{n}\right)$ by means of $\widetilde{T}\left(u_{n} \otimes x\right)=u_{n} \otimes T_{n} x$ for every $n \geq 1$ then

$$
F_{\widetilde{T}}(z)=\sum_{n=1}^{\infty}\left(u_{n} \otimes T_{n}\right) z^{n} .
$$


In other words, according to Theorem 5.9, $\left(T_{n}\right) \in\left(B_{1}(X), B_{q}(Y)\right)$ if and only if

$$
\sup _{\|x\| \leq 1|z|<1} \sup _{|z|}(1-|z|)\left\|\sum_{n=2}^{\infty}(n-1) n z^{n-2}\left(u_{n} \otimes T_{n} x\right)\right\|_{B_{q}(Y)}<\infty,
$$

which is clearly the same as

$$
\sup _{\|x\| \leq 1|z|<1} \sup _{|z|<\mid}(1-|z|)\left\|\sum_{n=1}^{\infty}(n+1) n z^{n}\left(u_{n} \otimes T_{n} x\right)\right\|_{B_{q}(Y)}<\infty .
$$

But

$$
\left\|\sum_{n=1}^{\infty}(n+1) n z^{n}\left(u_{n} \otimes T_{n} x\right)\right\|_{B_{q}(Y)}=\left(\int_{0}^{1} M_{q}^{q}\left(G_{x}, r|z|\right) d r\right)^{1 / q}
$$

where $G_{x}(z)=\sum_{n=1}^{\infty}(n+1) n\left(T_{n} x\right) z^{n}$.

¿From Lemma 5.6, $\left(T_{n}\right) \in\left(B_{1}(X), B_{q}(Y)\right)$ if and only if

$$
\sup _{\|x\| \leq 1} \sup _{0<r<1}(1-r)^{1+1 / q} M_{q}\left(G_{x}, r\right)<\infty \text {. }
$$

Since $G_{x}$ is the derivative of $E_{x}$ where $E_{x}(z)=\sum_{n=1}^{\infty} n\left(T_{n} x\right) z^{n+1}$, and then, Lemma 5.4 shows that the condition of being a multiplier is the same as

$$
\sup _{\|x\| \leq 1} \sup _{0<r<1}(1-r)^{1 / q} M_{q}\left(E_{x}, r\right)<\infty .
$$

Finally noticing that $E_{x}(z)=z^{2} F_{x}^{\prime}(z)$, and $M_{q}\left(E_{x}, r\right)=r^{2} M_{q}\left(F_{x}^{\prime}, r\right)$, now a look at Lemma 5.8 easily gives the final result.

Same proof as above gives the case $q=1$ in the previous theorem.

Theorem 5.11 Let $X$ and $Y$ be complex Banach spaces and $\left(T_{n}\right) \subset \mathcal{L}(X, Y)$. Then $\left(T_{n}\right) \in\left(B_{1}(X), B_{1}(Y)\right)$ if and only if

$$
\sup _{\|x\|=1} \sup _{0<r<1}\left(1-r^{2}\right) M_{1}\left(F_{x}^{\prime}, r\right)<\infty
$$

where $F_{x}(z)=\sum_{n=1}^{\infty} T_{n}(x) z^{n}$. 


\section{References}

[1] H. Amann Operator-valued Fourier multipliers, vector valued Besov spaces and applications, Math. Nachr. 186 (1997), 15-56.

[2] J.M. Anderson Coefficient multipliers and Sobolev spaces, J. Analysis, 1 (1993), 13-19

[3] J.M. Anderson, J. Clunie and C.Pommerenke On Bloch functions and normal functions, J. Reine Angew. Math., 270 (1974), 12-37.

[4] J. Berg and J. Lofstrom Interpolation spaces. An introduction, Springer-Verlag, Berlin and New York, 1973.

[5] J.L Arregui, O. Blasco, Bergman and Bloch spaces of vector valued functions, (to appear) 2001.

[6] O. Blasco, Spaces of vector valued analytic functions and applications, London Math. Soc. Lecture Notes Series 158, (1990), 33-48

[7] O. Blasco, Operators on weighted Bergman spaces and applications, Duke Math. J. 66 , (1992), 443-467.

[8] O. Blasco, Multipliers on weighted Besov spaces of analytic functions, Contemp. Math. 144 (1993), 23-33.

[9] O. Blasco, A characterization of Hilbert spaces in terms of multipliers between spaces of vector valued analytic functions, Michigan Math. J., (1995), 537-543.

[10] O. Blasco, Vector valued analytic functions of bounded mean oscillation and geometry of Banach spaces, Illinois J. 41 , (1997), $532-558$.

[11] O. Blasco, Multipliers on spaces of analytic functions, Can. J. Math. 47 (1995), 44-64.

[12] O. Blasco, Convolution by means of bilinear maps, Contemp. Math. 232 (1999), 85-103.

[13] O. Blasco, Remarks on vector-valued BMOA and vector-valued multipliers, Positivity 4 (2000), 339-356. 
[14] O. Blasco, Vector-valued Hardy inequality and B-convexity, Ark. Mat 38 (2000), 21-36.

[15] O. Blasco, Q. Xu, Interpolation between vector-valued Hardy spaces, J. Funct. Anal., 102, (1991), 331-359.

[16] P. Duren, $H^{p}$-spaces Academic Press, New York, 1970.

[17] P.L. Duren, B.W. Romberg, A.L. Shields Linear functionals on $H^{p}$ spaces, $0<p<1$, J. Reine Angew. Math., 238 (1969), 32-60.

[18] P.L. Duren and A.L. Shields Coefficient multipliers of $H^{p}$ and $B^{p}$ spaces, Pacific J. Math., 32 (1970), 69-78.

[19] T.M. Flett, On the rate of growth of mean values of holomorphic and harmonic functions, Proc. London Math. Soc., Vol 20, No3, (1976), 749-768.

[20] J. García-Cuerva, K.S. Kazarian, V.I. Kolyada, J.L. Torrea Vector-valued Hausdorff-Yooung inequality and applications, Russian Math. Surveys 53 (1998), 435-513.

[21] J.B. Garnett, Bounded analytic functions, Academic Press, New York, 1981.

[22] G.I. Gaudry, B.R.F. Jefferies and W.F. Ricker, Vector-valued multipliers. Convolution with operator-valued measures Dissertationes Mathematicae (2000).

[23] G.H. Hardy , J.E. Littlewood, Some properties of fractional integrals, II, Math. Z. 34 (1932), 403-439.

[24] M. Jevtic and I. Jovanovic, Coefficient multipliers of mixed norm spaces, Canad. Math. Bull. 36 (1993), 283-285.

[25] M. Jevtic and M. Pavlovic, Coefficient multipliers on spaces of analytic functions, Acta Sci. Math. (Szeged) 64 (1998), 531-545.

[26] C.N. Kellogg, An extension of Hausdorff-Young theorem,Michigan Math. J., 18 (1971), 121-127. 
[27] M. Mateljevic, M. Pavlovic, $L^{p}$-behaviour of the integral means of analytic functions, Studia Math. 77 (1984), 219-237.

[28] B. Maurey and G. Pisier, Séries de variables aléatories vectorielles indépendantes et propiétés géométriques des espaces de Banach, Studia Math., 58 (1976), 45-90.

[29] J. Peetre, Sur la transformation de Fourier des fonctions à valeurs vectorielles, Rend. Sem. Mat. Univ. Padova 42 (1969), $15-46$.

[30] A.L. Shields, D.L. Williams Bounded projections, duality and multipliers in spaces of analytic functions, Trans. Amer. Math. Soc., 162, (1971), 287-302.

[31] D. Vukotic, On the coefficient multipliers of Bergman spaces, J. London Math. Soc., 50 (1994), 341-348.

[32] L. Weis, Operator valued Fourier multiplier theorems and maximal regularity To appear.

[33] P. Wojtaszczyk, On multipliers into Bergman spaces and Nevalinna class, Canad. Math. Bull. 33 (1990), 151-161.

[34] K. Zhu, Operators on Bergman spaces, Marcel Dekker, Inc., New York, 1990.

[35] A. Zygmund, Trigonometric series,Cambrigde Univ. Press, New York, 1959.

Departamento de Análisis Matemático Universidad de Valencia

46100 Burjassot

Valencia

Spain

oblasco@uv.es
Dedpartamento de Matemáticas Universidad de Zaragoza 50005 Zaragoza Spain

arregui@posta.unizar.es 